\title{
The optical behaviour of ON 231 (W Comae) during and after the great outburst of spring $1998^{\star}$
}

\author{
G. Tosti ${ }^{1}$, E. Massaro ${ }^{2,3}$, R. Nesci ${ }^{2}$, S. Ciprini ${ }^{1}$, G. Nucciarelli ${ }^{1}$, M. Maesano ${ }^{2}$, F. Montagni ${ }^{2}$, C. M. Raiteri ${ }^{4}$, \\ M. Villata ${ }^{4}$, L. Lanteri ${ }^{4}$, and L. Ostorero ${ }^{5}$ \\ 1 Dipartimento di Fisica e Osservatorio Astronomico, Universitá di Perugia, via A. Pascoli, 00000 Perugia, Italy \\ 2 Dipartimento di Fisica, Università "La Sapienza", P.zle A. Moro 2, 00185 Roma, Italy \\ 3 Istituto di Astrofisica Spaziale, CNR, via del Fosso del Cavaliere 100, 00113 Roma, Italy \\ 4 Osservatorio Astronomico di Torino, Strada Osservatorio 20, 10025 Pino Torinese, Italy \\ 5 Dipartimento di Fisica Generale, Università di Torino, via P. Giuria 1, 10125 Torino, Italy
}

Received 5 February 2002 / Accepted 6 June 2002

\begin{abstract}
We present the results of the optical monitoring of the BL Lac object ON 231 from 1997 to the spring 2001, including the large outburst of spring 1998. After this event the mean source luminosity declined slowly and continued to show changes greater than one magnitude. A simple analysis of the variability time scales in the best-sampled segments of the light curve reveals that the luminosity changes were faster during the burst and slowed down in the subsequent years.
\end{abstract}

Key words. galaxies: active - galaxies: individual: ON 231 - galaxies: photometry

\section{Introduction}

The BL Lac object ON 231 (W Comae, 1219+285) has shown in the past a very remarkable activity, which has been studied in different bands of the electromagnetic spectrum, from radio to X-rays (Aller et al. 1999; Tosti et al. 1998; Massaro et al. 1999; Massaro et al. 2001; Tagliaferri et al. 2000). The historic optical light curve of this source (Massaro et al. 2001) shows that its mean luminosity increased after a minimum in the early seventies. In 1995 ON 231 started a phase of strong brightness and activity that culminated in a very strong outburst in April-May 1998, when it reached the record magnitude of $R=$ 12.2 (Massaro et al. 1999), comparable to that measured at the beginning of the past century when it was discovered as a variable star (Wolf 1916).

Photometric data, covering the period 1994-1997, i.e. when the source was very active but not as high as in 1998, are reported by Tosti et al. (1998, hereafter Paper I). In the following sections we present the results of our photometric observations of ON 231, including the great outburst of spring 1998 and the subsequent period up to June 2001, and compare them with the source behavior in other epochs. No other events comparable to that of 1998 have been observed indicating that it is quite uncommon and must be related to relevant changes of the inner structure of the source.

Send offprint requests to: $\mathrm{G}$. Tosti, e-mail: gino.tosti@pg.infn.it

* Table 2 is only available in electronic form at the CDS via anonymous ftp to cdsarc.u-strasbg.fr $(130.79 .128 .5)$ or via http://cdsweb.u-strasbg.fr/cgi-bin/qcat?]/A+A/395/11

\section{Observations and data reduction}

The photometric data presented in this paper have been obtained in the course of a monitoring program of a sample of bright blazars carried out since 1994 by our collaboration. The telescopes used were the AIT $(0.40 \mathrm{~m})$ of the Perugia Observatory, the $1.05 \mathrm{~m}$ astrometric telescope of the Observatory of Torino, the TRC $70(0.70 \mathrm{~m}, f / 8.3)$ of the IAS-CNR and "La Sapienza" University, the reflector telescope $(0.50 \mathrm{~m}, f / 4.5)$ of the Astronomical Station of Vallinfreda near Rome and another reflector $(0.35 \mathrm{~m})$ located near Greve, in Tuscany. All the instruments were equipped with CCD cameras and standard filters for the Johnson $B, V$ and Cousins $R, I$ bandpasses. Bias, dark, flat field correction and data reduction of the frames were performed with IRAF tasks, and other locally developed software. Aperture photometry was made with IRAF-apphot or equivalent algorithms and using a sequence of reference stars in the same field of ON 231. The $V, R, I$ magnitudes of these stars are given in Fiorucci \& Tosti (1996), while the $B$ values can be found in Paper I.

All the photometric data with the corresponding uncertainties, from March 1997 to June 2001 are listed in Table 2, available in electronic form at the CDS, and update those given in Paper I. The codes PG, RM, TO, indicate the Perugia, Roma, and Torino telescopes, respectively.

\section{The optical variability}

The 1997-2001 light curves in the $V$ and $R$ bands, those with the densest sampling, are shown in Fig. 1. Table 1 gives 


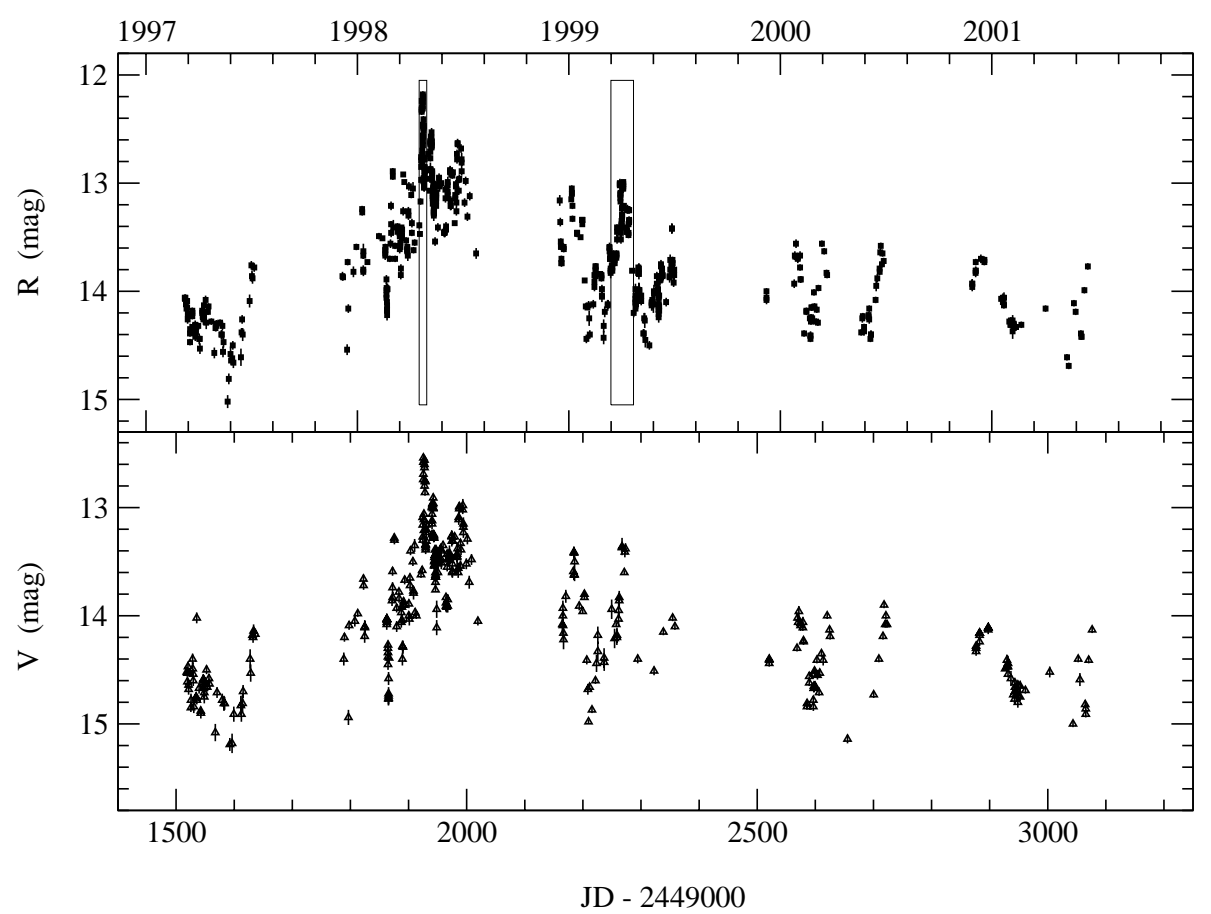

Fig. 1. The light curve of ON 231 in the $R$ (upper panel) and $V$ (lower panel) bands in the period March 1997-June 2001. In the upper panel the data in the two rectangular boxes around JD 1926 (April 1998) and JD 2270 (March 1999) mark the segments of the light curve plotted on expanded time scales in Fig. 2.

a summary of the whole campaign (1994-2001) and reports, for each visibility period of the source, the range of measured magnitudes and the number of observations (in parenthesis) for each filter. Form the light curve the relevance of the 1998 outburst and the subsequent decline phase it is quite clear. The two best sampled seasons are those of 1998 and 1999, while in the years 2000 and 2001 unfavorable meteorological conditions limited the observing window.

It is evident that the typical source behavior was quite similar to that observed in the years before the burst (see Paper I) with variations larger than 1 mag.

An important subject in the study of BL Lac objects is the estimate of the variability time scales and their relation to the amplitude of brightness changes and, more generally, with the state of the source. This is not a simple task because of the limited and not regular sampling, although our light curves are likely the richest available data set. We therefore analyzed only a few episodes reasonably well described in our data series. For the sake of homogeneity one has to distinguish between the rising and decaying parts of a given event: different physical processes like injection, radiative cooling and escape of the emitting electrons, having different typical time scales (see for instance the discussion in Massaro et al. 1996), could be in fact responsible for such differences. In our light curves the number of well sampled rising branches is by chance greater than that of decaying ones, and therefore we focused our analysis only on the former type.

From a simple inspection of the light curves one can see that in 1997 the $R$ magnitude showed an oscillating behaviour between 14.0 and 14.5 for about two months, and after it declined to 15 to brighten to about 13.7 in about 40 days.
The observational seasons of 1998 and 1999 are those with the finest sampling. In the former ON 231 showed a mean brightening trend culminating in the great outburst of April 1998: before the flare three well-sampled rising branches can be found with magnitude changes of $0.60,0.86$ and 1.25 occurring in the rather short times of 2, 4 and 10 days. During the main burst a large and rapid variability was observed (Massaro et al. 1999) with a change of $0.6 \mathrm{mag} /$ day that produced a double peak structure (Fig. 2, upper panel). In 1999 the source luminosity remained high and varied between 13.0 and 14.5: in particular we observed another burst having a time structure quite similar to that of the strong event of 1998, but evolving on time scales longer by a factor of about 3 , as shown in the lower panel of Fig. 2. Some rising branches of these two events were also used to estimate the time scales and the corresponding magnitude changes. Of course we do not mean that these particular time profiles represent a stable or a recurrent characteristic of the behavior of ON 231; they are only an indication of how the variability time scales change in different source's states.

It is also interesting to compare the recent activity of ON 231 with that before the large burst. The photometric data given in Paper I show that in 1994 the source was not very bright and its $R$ magnitude was never smaller that 14.5 . In 1995 and 1996 the behaviour of ON 231 was more similar to that observed after 1999: the $R$ magnitude varied in the interval 13.4-14.5 with large flares evolving over time scales of months.

We also performed a comparison among the rising time scales and the corresponding variation amplitudes as observed at various epochs from 1995 to 2000 . To this end we selected only well-sampled events that allowed a rather safe estimate of 
Table 1. Summary of the optical monitoring (1994.03.05-2001.06.14).

\begin{tabular}{lllll}
\hline \hline Obs. Period & $B$ & $V$ & $R$ & $I$ \\
\hline $1994-1995$ & $14.23-15.77(61)$ & $13.73-15.30(98)$ & $13.36-15.00(113)$ & $12.90-14.43(86)$ \\
$1995-1996$ & $14.29-15.67(42)$ & $13.61-15.26(176)$ & $13.38-14.74(137)$ & $12.95-14.03(111)$ \\
$1996-1997$ & $14.26-15.51(34)$ & $13.86-15.19(133)$ & $13.52-14.81(146)$ & $13.02-14.22(98)$ \\
$1997-1998$ & $13.00-15.09(66)$ & $12.54-14.94(170)$ & $12.18-14.54(204)$ & $11.81-13.89(167)$ \\
$1998-1999$ & $13.74-15.25(34)$ & $13.36-14.44(37)$ & $13.00-14.5(171)$ & $12.49-13.94(92)$ \\
$1999-2001$ & $14.27-15.41(16)$ & $13.96-14.84(62)$ & $13.56-14.44(78)$ & $13.08-13.95(60)$ \\
\hline
\end{tabular}
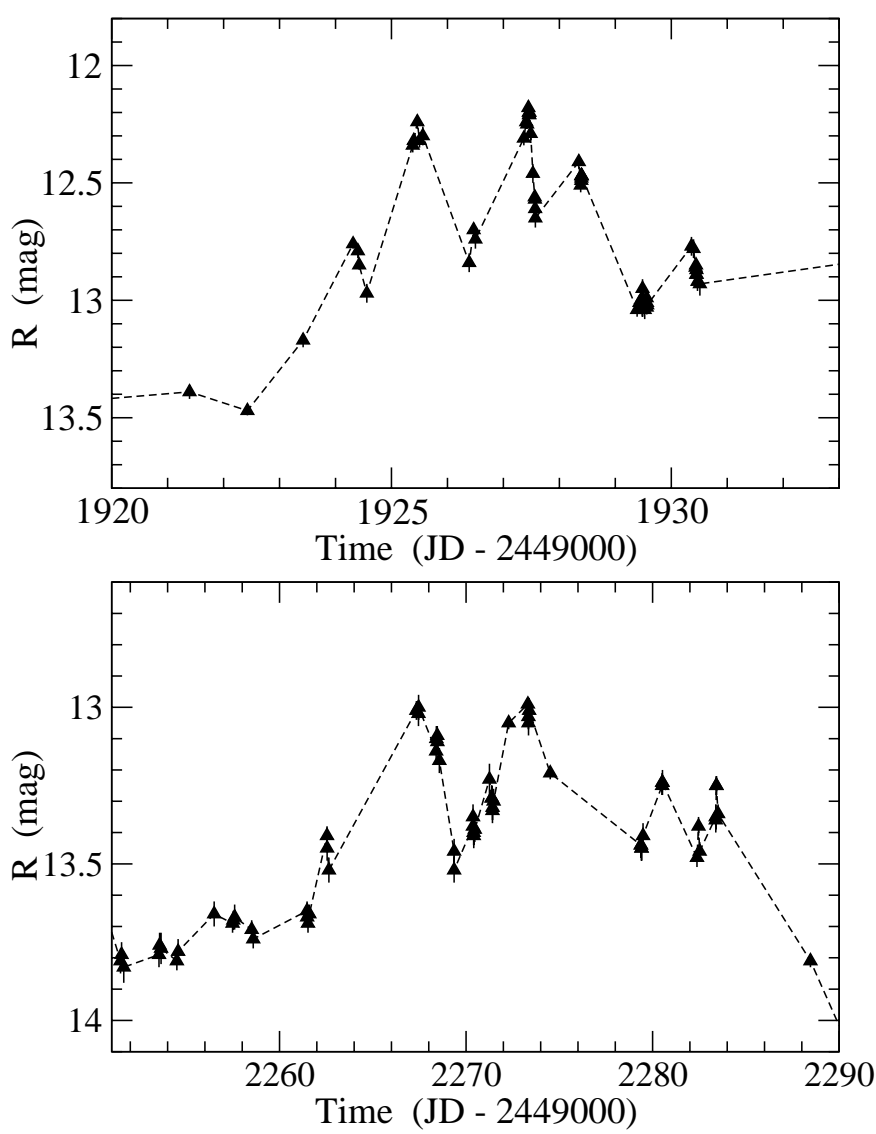

Fig. 2. Comparison between two portions of the light curve of ON 231 in the $R$ filter: during the outburst of spring 1998 (upper panel, $1920=1998$ April 16) and in 1999 (lower panel, $2260=1999$ March 22). The duration of the former event is a factor of 3 shorter than that of the latter, while the ratio of the magnitude scale is $4 / 3$.

these parameters, and also those with a faster variation rate. The time scale uncertainties are not statistical but correspond to half of the time distance with the nearest data. The resulting values are shown in Fig. 4: the points are apparently sparse without a regular pattern, however if they are grouped according to particular periods some regular trends appear. The points corresponding to the most rapid and largest variations are, of course, those of the 1998 outburst, while those of only a few months before approximately follow the same trend, but are shifted towards longer time scales. A similar slope is also apparent for the points of other years, but with the exception of those corresponding to the shortest variations (say shorter than 2 days) that are nearly aligned with the 1998 not-outburst points.

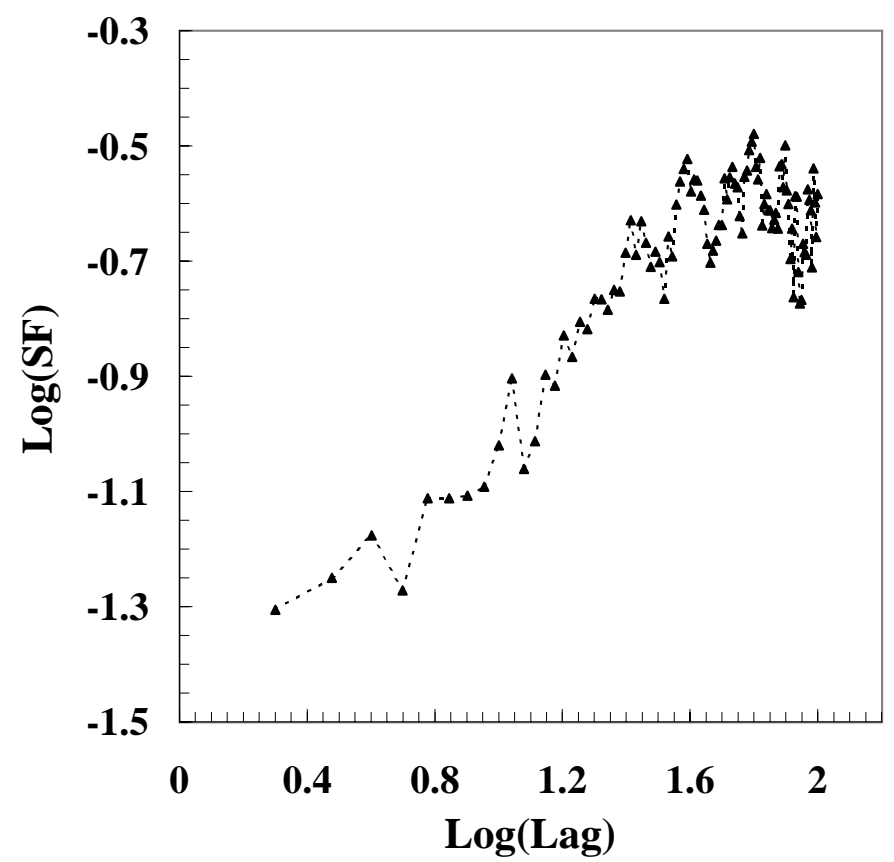

Fig. 3. The structure function of the 1994-2001 $R_{\mathrm{c}}$ light curve.

\section{Discussion and conclusions}

The recent optical activity of ON 231 has been intensively monitored since 1994: multiband photometric data for the period 1994-1997 are reported in Paper I, and in the present work we update these light curves to June 2001. In this period ON 231 underwent a very active phase and reached the highest luminosity seen in the last several decades.

In order to investigate the nature of the source variability we analyzed the entire 1994-2001 data set in the $R_{\mathrm{c}}$ band using the Structure Function (SF) technique (see e.g. Haufnagel \& Bregman 1992; Paltani et al. 1997). We recall that a SF analysis is similar to a power spectrum analysis and if the mean trend of the SF can be described by power law, say $S F \propto t^{\alpha}$, then the Fourier power spectrum $P(f) \propto f^{-(\alpha+1)}$, where $f$ is the frequency. The SF of the 1994-2001 $R_{\mathrm{c}}$ light curve, obtained using 1 day binning in the range 1-100 days, is reported in Fig. 3. The slope $\alpha$ of the $\log (S F)$ versus $\log$ (timelag) is $\alpha=0.53$, a value intermediate between that expected for a pure shot noise spectrum $(\alpha=1)$ and pure flickering $(\alpha=0)$. This result confirms the complex nature of the source variability. The $\alpha$ value we found is close to the value of $\alpha=0.67$ typical of fully developed turbulence, suggesting the possibility 


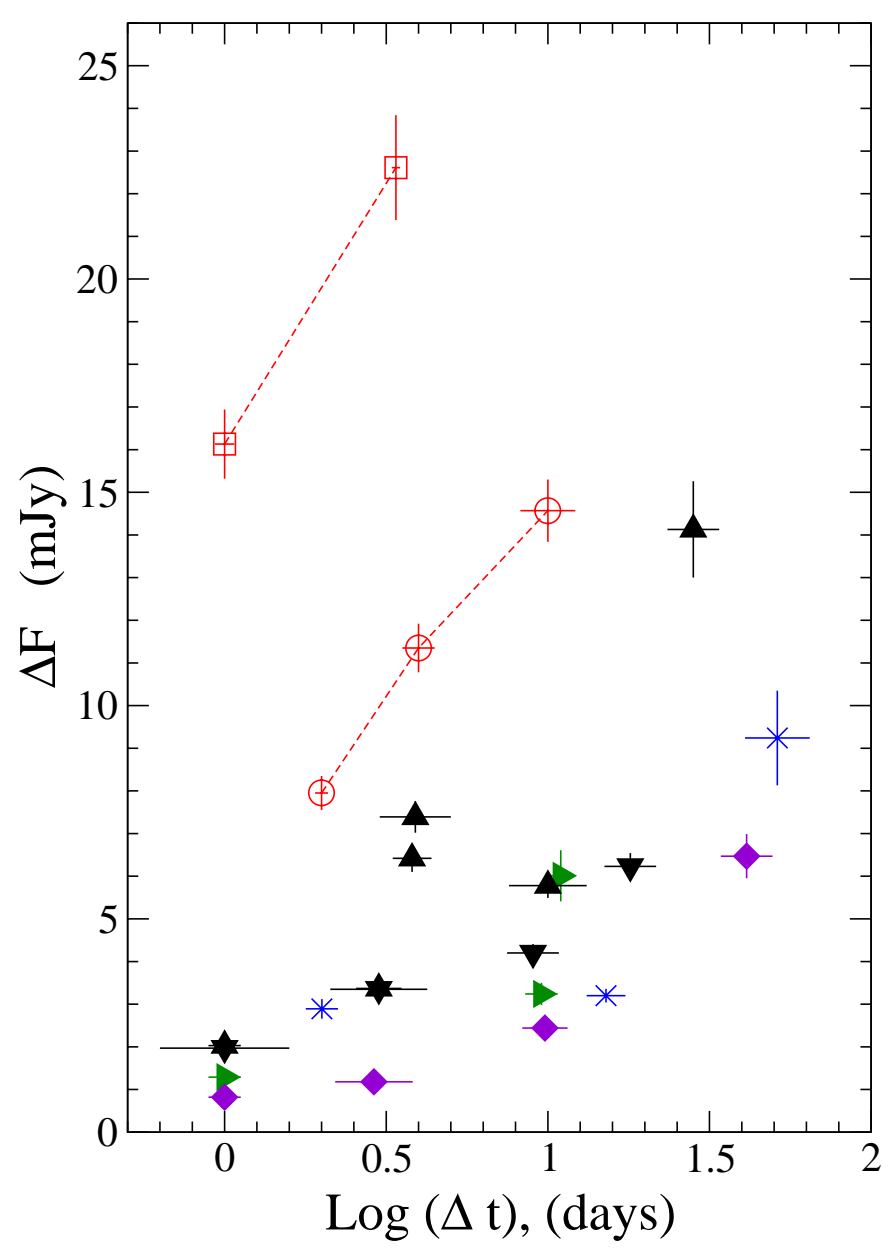

Fig. 4. The relation $\Delta F$ vs. $\log (\Delta t)$ for the rising branches of some well sampled events: 1995 (crosses), 1996 (triangles right), 1997 (filled diamonds), 1998-before flare (open circles), 1998-flare (open squares), 1999 (triangles up) and 2000 (triangles down).

that the ON 231 variability could be a related to fluctuations of the magnetic field or of the bulk motion velocity of the emitting region inside the jet.

We furthermore tried to obtain some information on the variability times scales in different states of the source. This analysis is not simple because of the irregular data sampling and must be limited to particular segments. It is general wisdom that time scales are longer when the amplitudes of variations are greater (see e.g. Ulrich et al. 1997). We verified this behavior for $\mathrm{ON} 231$, for some rising branches, but it was also observed for the decays. The results of Fig. 4 show that the relation $\Delta F$ vs. $\log (\Delta t)$ changed with the states of the source, in the sense that variations are faster when the source is more active. Changes over scales of two days or shorter and with an amplitude of a few tenth of magnitude, however, are observed in all luminosity states. This finding can be explained if the long and short variations are due to different components, and one being responsible for the changes of the mean brightness of the source over times scales of years and the other responsible for faster changes (time scales of weeks or days).

This result, if confirmed by new data either on ON 231 or other similar objects, offers an interesting clue about the origin of the luminosity changes, at least for those caused by the component responsible for the slow and large amplitude variations. The luminosity of a synchrotron source depends upon three main parameters: the magnetic field $B$, the energy $\left(\gamma m c^{2}\right)$ and the number $N$ of radiating electrons. A change of $N$ can be excluded because it implies a proportional variation of the luminosity but not increase of the time scale, unless one admits that the acceleration and the injection of a larger number of electrons are briefer when this number increases. In a similar way an increase of $B$ and/or $\gamma$ would reduce the radiative cooling time proportional to $1 / \gamma B^{2}$, but it would not directly affect the rising time scale. According to the standard model for BL Lac sources (originally proposed by Blandford \& Rees 1978) we are observing a jet pointing with a small angle, $\theta$, to the line of sight. The observed time scales are reduced by the beaming factor $\delta=1 / \Gamma(1-\beta \cos \theta)$, where $\beta$ and $\Gamma$ are the bulk velocity and Lorentz factor of the emitting region inside the jet, while the apparent flux is increased by a higher power of $\delta$. A regular change of this factor can be then a simple interpretation of the relation shown in Fig. 4.

A variation of the beaming factor, due to a change of $\theta$, produced for instance by the approaching of the innermost jet direction to the line of sight, could be responsible for the long-term trend of the mean luminosity. In the case of ON 231 the minimum distance was likely reached between the autumn of 1997 and spring of 1998 when the source was at the maximum brightness and showed the most rapid variability. This interpretation agrees with recent VLBI observations (Massaro et al. 2001) performed just after that period which showed the onset of a new bright component emerging from the nucleus in direction opposite to that of the jet. Under the simple assumption that $\beta$, and consequently $\Gamma$, remains practically constant, one can compute the relation between the values spanned by $\theta$ in our observations. If $\delta_{\mathrm{M}}$ is the maximum value of the beaming factor, corresponding to the jet direction $\theta_{\mathrm{M}}$, the change of the time scale by a factor of about 3 , as found above, implies the relation:

$\delta_{\mathrm{M}} / \delta=(1-\beta \cos \theta) /\left(1-\beta \cos \theta_{\mathrm{M}}\right) \simeq 3$

and from this follows

$\cos \theta_{M}=\frac{1}{3 \beta}(2+\beta \cos \theta)$.

For a value of $\beta=0.995$, corresponding to $\Gamma=10$, we found that a change of this angle, for instance from about $5^{\circ}$ to about $12^{\circ}$ (of course other values are possible) can account for the different time scales observed during the maximum of April 1998 and in 1999.

If this scenario is correct, in the next years one could expect that the jet direction will continue to move away from the line of sight implying a slow fading of the mean apparent luminosity of ON 231 together with an increase of the typical variability time scales. Of course this model is not the only way to explain the behavior of ON 231. Other models, like for instance a binary system of massive black holes (Lehto \& Valtonen 1996) proposed for long term periodicity of OJ 287 , can also be used to interpret these light curves. The developing of observational programs for multifrequency monitoring, possibly with worldwide participation, is therefore one of 
the most useful strategies to confirm or disprove the proposed interpretation.

Acknowledgements. This work was performed with the financial support by Italian Ministry of University and Scientific Research under the grants Cofin 98-02-32 and Cofin 2001/028773.

\section{References}

Aller, M. F., Aller, H. D., Hughes, P. A., \& Latimer, G. E. 1999, ApJ, 512,601

Blandford, R. D., \& Rees, M. J. 1978, in Pittsburgh Conf. on BL Lac Objects (Univ. of Pittsburgh), 328
Fiorucci, M., \& Tosti, G. 1996, A\&AS, 116, 403

Hufnagel, B. R., \& Bregman, J. N. 1992, ApJ, 386, 473

Lehto, H. J., \& Valtonen, M. J. 1996, ApJ, 460, 207

Massaro, E., Nesci, R., Maesano, M., et al. 1996, A\&A, 314, 87

Massaro, E., Maesano, M., Montagni, F., et al. 1999, A\&A, 342, L49

Massaro, E., Mantovani, F., Fanti, R., et al. 2001, A\&A, 374, 435

Paltani, S., Courvoisier, T. J.-L., Blecha, A., \& Bratschi, P. 1997, A\&A, 327, 539

Tagliaferri, G., Ghisellini, G., Giommi, P., et al. 2000, A\&A, 354, 431

Tosti, G., Fiorucci, M., Luciani, M., et al. 1998, A\&AS, 130, 109

Ulrich, M., Maraschi, L., \& Urry, C. M. 1997, ARA\&A, 35, 445

Wolf, M. 1916, Astron Nachr., 202, 415 